\title{
Organizzazione e utilizzazione di un programma di analisi spettrale
}

\author{
(Organization and use of a program of spertral analysis)
}

\author{
F. MaT'THOLI (*)
}

Ricevuto il 10 Settembre, 1975

Riassunto. - Si riassumono diversi problemi, sia teorici che pratici, inerenti lanalisi auto e cross spettrale delle serie temporali, per porro in evidenza lo spirito con il quale tali tecniche vanno impiegate.

Si raggiungono poi conclusioni circa i criteri secondo i quali un procramma di analisi spettrale dovrebbe essere organizzato ed utilizzato.

SUmmar. - Several theoretical and practical problems on spectral and cross spectral analysis of time series are summarized in oreler 10 point out the way in which these techniques are to be emploved.

Conclusions are drown about the criterions to be followed in the organization and use of a program of spectral analysis.

\section{1. - INTRODUZIONE}

I programmi di analisi spettrale che si trovano in commercio non sono, di solito, ampiamente utilizzati. In genere un utente o si costruisce da sé il suo programma, oppure utilizza un programma costruito da altri, continuando però ad applicarlo alla stessa ristretta catecroria di problemi per i quali questo era stato originariamente pensato.

La maione di questo stato di cose risiele nella convinzione, comunemente accettata, che l'analisi spettrale sia un ben preciso susseguirsi di determinate operazioni che, applicate alla serie da esaminare, proBologna.

$\left(^{*}\right)$ Assegnista Ministeriale presso l'Istituto di Fisica "A. Righi", di 
duce come risultato le funzioni spettrali desiderate. Questa erronea visione delle cose ha indotto molti programmatori a costruire dei programmi estremamente rigidi e, di conseguenza, difficilmente adattabili alle esigenze dei diversi utenti.

Per chiarire meglio la natura del problema proposto facciamo un esempio in cui esso, al contrario, non esiste. Se si vuole invertire una matrice $\dot{e}$ facile usare il sottoprogramma relativo: basta infatti definire pochi parametri e riservare un'opportuna area di memoria. Poiché l'operazione che si vuole eseguire è univocamente determinata, ne segue che l'applicazione del programma è del tutto automatica: l'utente non deve, nó può, formulare alcuna decisione su di essa.

Per l'analisi spettrale invece la situazione è differente: ci sono infatti diverse ragioni che impediscono di utilizzare un qualunque programma di analisi spettrale senza una previa discussione ed una susseguente continua interazione con esso da parte dell'utente.

Cerchiamo di riassumere i diversi problemi che portano a questa importante conclusione.

\section{2. - Problemi connessi ald'anaLisi spettralle}

2.1. - Ma un punto di vista statistico una qualunque funzione spettrale è caratterizzata da una certa stabilità er una certa risoluzione in frequenza, dipendenti amberlue da uno stesso parametro, il numero di gradi di libertà del sistema (Blackman and Tuckey, 1958; Jenkins and Watts, 1969) $(1,2)$.

Se gli spettri sono "smooth ", allora è conveniente tenere alto tale parametro, ottenendo così un'altra stabilità, unita però arl una bassa risoluzione in frequenza; se invece negli spettri compaiono diversi picchi stretti e vicini, è conveniente usare un basso numero di gradi di libertà, per essere in grarlo di risolverli in maniera sodllisfacente, anche a scapito della valutazione della loro ampiezza, resa difficile dalla bassa stabilità così ottenuta $\left({ }^{2}\right)$.

Come si vede, la definizione di questo parametro dipende dal risultato finale che si otterrà. Sarà quindi necessario stabilirlo dapprima in base alle considerazioni teoriche che di volta in volta si potranno portare, e poi ricorregerlo in base alle indicazioni spettrali ricavate dal primo tentativo. In generale un calcolo spettrale deve essere fatto con 2-3 diversi numeri di grarli di libertà, per poter stabilire, dall'evoluzione 
delle stime da un numero di gradi di libertà all'altro, la forma vera degli spettri da valutare $\left({ }^{2}\right)$.

In alcuni casi la procedura può anche essere più complessa. Se si sospetta che nelle serie originali siano contenute delle componenti periodiche di frequenza fissa, oppure delle componenti stocastiche di larghezza di banda molto ristretta, è necessario eseguire il calcolo degli spettri con 2 gradi di libertà. In conseguenza del risultato ottenuto si può decidere di eliminare quelle eventuali componenti, per esempio mediante filtraggio, oppure di lasciarle, tenendone però conto nell'interpretazione degli spettri finali.

Analogamente, quando uno spettro presenta un andamento molto complesso, può essere conveniente filtrare le serie originali in più parti, in modo che la loro somma dia ancora le serie originali, ed eseguire l'analisi spettrale di ogni parte separatamente dalle altre, con dei numeri di gradi di libertì di volta in volta differenti $\left(^{2}\right)$.

In alcuni casi, quando gli spettri hanno delle forme molto regolari, può essere preferibile usare la cosiddetta tecnica del "prewhitening", che consiste nell'elaborare le serie originali opportunamente filtrate, in morlo che gli spettri risultanti abbiano un andamento per quanto possibile costante; conoscendo poi la funzione di risposta del filtro usato, si può risalire alla forma degli spettri delle serie originali, compiento errori di calcolo di minor entità $\left(^{1}\right)$.

In definitiva possiamo affermare che una valutazione spettrale viene raggiunta attraverso un processo iterativo, che può richiedere in alcuni casi delle operazioni intermedie di filtraggio.

2.2. - Esiste inoltre un'altra eategoria di problemi, legata alla possibilità di valutare le stime spettrali passando attraverso il calcolo delle funzioni di covarianza o, più direttamente, attraverso il calcolo della trasformata di Fonrier (veloce) delle serie originali. Liutente deve quindi valutare preventivamente quale delle due vie si arlatta meglio al suo problema.

In genere si sceglie la prima via tutte le volte che non sia indispensabile seguire la seconda. Infatti le funzioni di covarianza hanno di per sé un loro significato che si richiama direttamente alla teoria in base alla quale le funzioni spettrali sono state introdotte. Esse permettono di individuare eventuali ritardi fra una serie e l'altra, nonchí la presenza o meno di spiccanti andamenti periodici.

Dalla valutazione del massimo dei ritardi per cui esse sono significativamente diverse da zero si puó anche ricavare la risoluzione in 
frequenza più opportuna da tenere nel calcolo spettrale seguente. Qualora poi una funzione di cross covarianza presenti un picco in prossimità dell'origine, traslarla in morlo che il picco sia centrato nell'origine prima di effettuarne la trasformata di Fourier, produce delle stime spettrali migliori ( $\left.{ }^{2}\right)$.

Bisogna ricordare che le operazioni che richiedono il maggior tempo di calcolo sono in primo luogo le funzioni di covarianza, calcolate col metodo diretto, e in secondo la trasformate di Fourier, qualora non si impieghi un metodo di Fast Fourier Transform (F.F.T.).

Nel caso però in cui, pur avendo scelto la seconda strada, si sia comunque interessati alle funzioni di covarianza bisogna ricordare che queste possono essere calcolate come antitrasformate di Fourier delle funzioni spettrali, anche se i risultati cosi ottenuti non sono molto buoni.

Le funzioni di covarianza d'altra parte possono essere ottenute direttamente dalle serie originali attraverso l'uso ripetuto di un programma di F.F.T.: anzi, se si è interessati alla lor o valutazione per ritardi molto grandi, questa è l'unica soluzione possibile. Gli spettri a loro volta potrebbero essere calcolati anche a partire clalle funzioni di covarianza cosi ottenute. Bisogna però notare che questo metodo, anche se non richiede tempi di calcolo eccessivi, esige l'mpiego di una notevole quantità di memoria ausiliare.

2.3. - Aceanto ai problemi citati che sono strettamente legati, i primi alla stessa natura teorica clell'analisi spettrale, e i secondi ai diversi metorli di calcolo numerico esistenti, ne esistono altri ancora, legati più alle esigenze specifiche dei diversi utenti, che riguardo prevalentemente le operazioni di lettua e di prima trattazione dlei clati, nonché i diversi modi in eui i risultati finali possono essere presentati in uscita.

Ise operazioni di lettura si divessificheranno a seconda cle i dati si trovino su scheda, su nastro o altro. Può darsi poi il caso che nelle serie originali manchi una certa quantità dli dati, in intervalli di varia lunghezza e variamente disposti.

Questo problema può essere affrontato seguendo una delle due seguenti vie: o si sostituiscono ai dati mancanti clei valori calcolati in base ai rlati a disposizione, mediante opportune formule interpolatorie, oppure si alottano per il calcolo delle funzioni di covarianza formule particolari, che tengono conto solo dei dati presenti. La prima soluzione è indispensabile se si deve applicare direttamente alle serie in esame un programma di F.F.T., ed è da evitare per quanto possibile, poiché 
qualunque sia l'operazione di interpolazione adottata, si introduce nelle serie un'informazione arbitraria, i cui effetti sono difficilmente preverlibili. Isa seconda soluzione consiste nel calcolare le funzioni di covarianza mediando soltanto sui prodotti eseguibili fra le serie originali e le serie ritardate (qui per prodotto eseguibile si intencle un prodotto in cui amberlue i termini siano dati noti). Le stime che cosi si ottengono avrammo un minore numero di grarli di liberti, rispetto al caso in cui tutti i dati fossero noti, vista l'inevitabile perdita di informazione, e saramo fra di loro maggiormente correlate; esse però saranno "unbiased", come conseguenza del fatto che non è stata immessa aleuna informazione aggiuntiva (Mattioli; Mattioli, Tibaldi and Pellizzarr(i) $(3,4)$.

Mentre la scala su cui porre le funzioni di covarianza non può altro che essere lineare, non così si può dire per le funzioni spettrali. Poiché i logaritmi delle funzioni autospettrali e le arcotangenti iperboliche delle coerenze quarlrate possierlono intervalli di confidenza costanti, spesso si possono trovare quelle grandezze in quoste scale. Tutte le volte che le più forti variazioni spettrali avvengono nel campo delle basse frequenze, può essere opportuno arlottare una scala logaritmica in ascisse, cosi come la scala doppio logaritmica è particolarmente arlatta per rivelare degli andamenti di potenza. Una scala caratteristica delle stime autospettrali è poi quella che dispone in ascissa il logaritmo della frequenza $e$ in ordinata il prodotto della funzione spettrule per la frequenza: ma tale scala permette di avere un grafico in cui l'area sottesa dalla curva con l'asse delle ascisse mantiene, come nel easo di scala doppio lineare, il significato di potenza relativa a quel campo di frequenza. A questo punto però è bene osservare che gli spettri possono essere posti con una scala logaritmica nella frequenza, solo nell'ipotesi che siano stati calcolati con il metodlo della F.F.T.

Inoltre possono rendersi necessarie tante operazioni preliminari dei dati, come istogrammi, medie progressive, normalizzazioni, ecc. ..., che, se pur non indispensabili, fa però molto comodo trovare accanto ad un programma di analisi spettrale.

2.4. - Esiste insomma una notevole quantità di problemi che non permettono né di pensare all'analisi spettrale come ar un susseguirsi ben determinato di operazioni, né di utilizzare un programma di analisi spettrale come un sistema che da un ingresso costituito dalle serie originali, fornisce una o più uscite, in un ordine sequenziale prefissato a priori, corrispondenti ai risultati voluti. 


\section{3. - ORgaNizzazIONE DI UN PROGRAMMIA DI ANALISI SPETTRALE}

Se la bontà di un generico programma, come quello per l'inversione di una matrice viene solitamente valutata in base al tempo di calcolo e alla memoria richiesti per eseguir e le operazioni volute, questi fattori risultano di secondaria importanza in un programma di analisi spettrale. Le differenze di tempo di calcolo e di memoria usati dipendono prevalentemente dal metolo scelto, e tutte le possibili varianti dello stesso metodo introducono cambiamenti di ben scarso rilievo. Il fattore determinante che puó indurre un utente ad adottare un certo programma di biblioteca è la possibilità di ritrovare in esso fra tutte le operazioni che abbiamo precedentemente citato, quelle che gli servono per il suo problema particolare. Quindi ciò che bisogna fornire all'utente non è un unico programma, ma un complesso di sottoprogrammi, ciascuno dei quali esegua un insieme elementare di operazioni, costruiti in modo da poter essere semplicemente connessi l'uno all'altro secondo la necessità.

Una possibile suddivisione in blocchi elementari delle diverse operazioni che intervengono nell'analisi spettrale è la seguente:

1) Lettura dei dati.

2) Operazioni preliminari (interpolazioni di dati mancanti, normalizzazioni, ecc. ...).

3) Filtraggio dei dati.

4) Calcolo delle funzioni di covarianza.

5) Calcolo delle funzioni di covarianza attraverso l'uso ripetuto della F.F.T.

6) Calcolo delle funzioni auto, co- e q-spettrali come trasformate di Fourier delle funzioni di covarianza.

7) Calcolo delle funzioni, auto, co- e q-spettrali col metodo F.F.T.

8) Calcolo delle funzioni cross spettrali derivate (guadagno, rumore, coerenza e fase) a partire dalle funzioni cross spettrali semplici (auto spettri, co-e q-spettri).

9) Messa in scala opportuna delle grandezze di uscita, con relativo calcolo degli intervalli di confidenza.

10) Stampa.

Nella figura 1 poniamo in rilievo i più comuni tipi di connessione di questi blocchi elementari. 


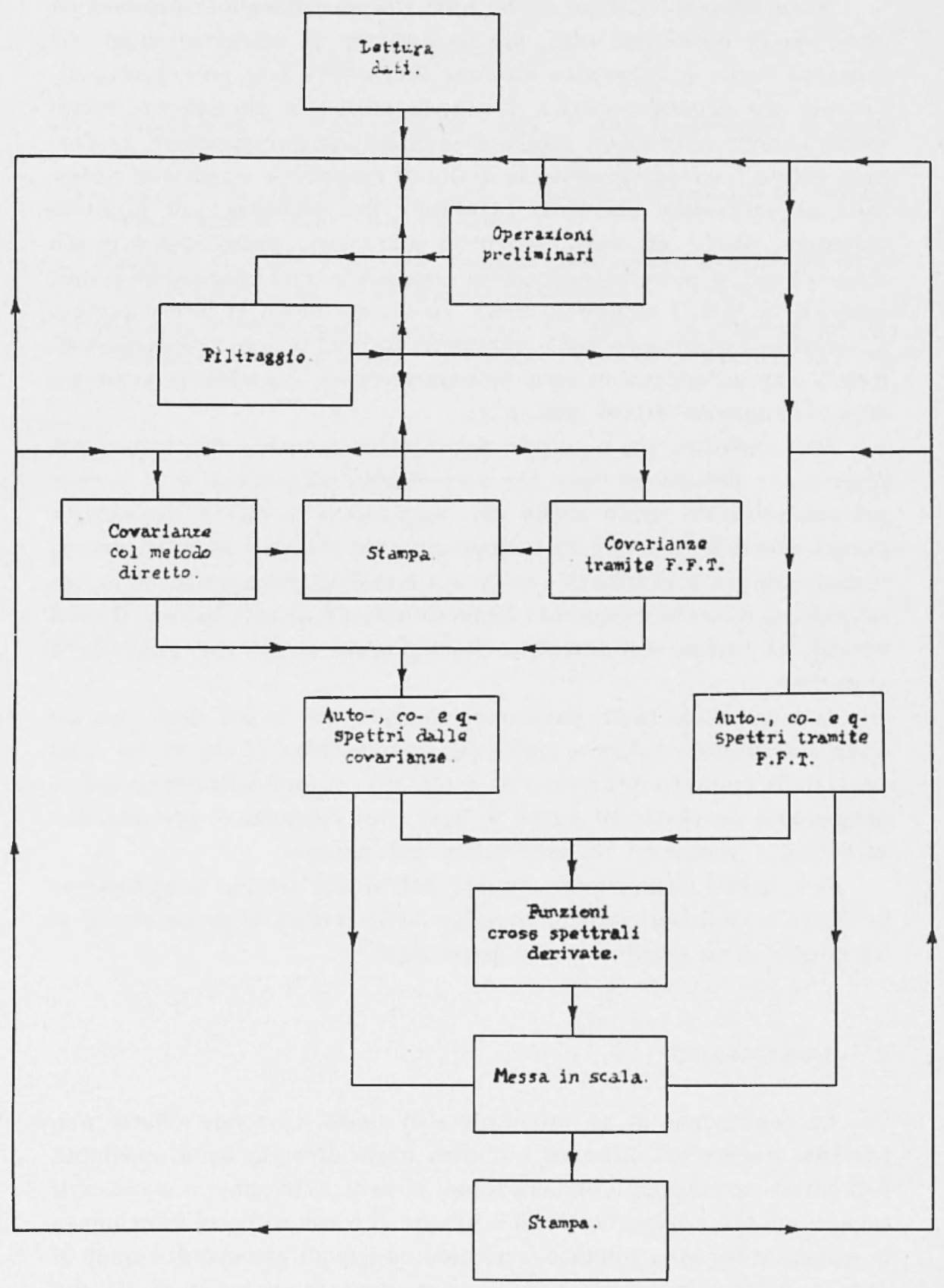

Fig. 1 - Schema a blocchi delle principali operazioni relative all'analisi spettrale. 
Naturalmente esistono anche altri tipi di comnessioni possibili ed altri tipi di operazioni utili, ma un insieme di sottoprogrammi che eseguisse tutte le operazioni elencate lasciarebbe ben poco lavoro all'utente che esigesse qualcosa di più. In pratica il suo compito consisterebbe nello scrivere un programma principale per chiamare, nell'ordine voluto i sottoprogrammi in grado di eseguire le operazioni necessarie al particolare problema in esame. Per renderne più facile la chiamata, poiché gli stessi parametri compaiono molto spesso in più d'uno di essi, si possono trasferire in "common" tutti i parametri scalari esistenti in tutti i sottoprogrammi. In questo morlo la loro chiamata avverrebbe esplicitando come parametri formali le sole grandezze vettoriali, che all'interno di ogni sottoprogramma dovrebbero avere un dimensionamento fittirio pari a 1 .

Per agevolare poi il calcolo del dimensionamento dei vettori nel programma principale, visto che esso cambia notevolmente al variare del procedimento usato anche per raggiungere lo stesso risultato, ̀̀ sempre possibile costruire un sottoprogramma che, in base ai parametri scalari, sempre trasferiti in "common" e refiniti precerlentemente, sia in grarlo di dare come risposta i dimensionamenti da attribuire ai cliversi vettori, al variare dei singoli sottoprogrammi in cui essi potrebbero apparire.

Infine, poiché molti parametri vengono per lo più usati con un certo determinato valore, e anche per evitare errori di esecuzione derivanti dalla mancata definizione di uno di essi, si può costruire un sottoprogramma da chiamare prima di ogni altra operazione, per inizializzare tutti i parametri col loro valore più comune.

Con questi strumenti il compito dell'utente sarebbe grandemente facilitato e, in refinitiva, consisterebbe nella stesura di poche decine di istruzioni, salvo esigenze molto particolari.

\section{4. - Conclusioni}

Ia costruzione di un programma di analisi spettrale siffatto non presenta particolari difficolti e d'altra parte dipende dalla sensibiliti dell'autore aggiungere o togliere alcuni blonchi, sviluppare o meno certi settori: ciò che è importante, ed è quanto si è voluto porre in evidenza in questa sede, sono soltanto le ragioni ed i modi attraverso i quali si deve e si può salvaguardare in un programma ri analisi spettrale una struttura che permetta all'utente un "dialogo" con esso. 


\section{BIBLIOGRAFIA}

(') Blackmax R. B., Tuckey J. W., 1958. - The Measurement of Power Spectra. "Dover Publications", Inc., New York.

$\left(^{2}\right)$ I ENkins C. M., Watts D. G., 1969. - Spectral Analysis and its Applications. "Holden Day", San Francisco.

${ }^{3}$ ) Mattioli F., 1975. - Spectral Analysis of Wind and $\mathrm{SO}_{2}$ Concentration in the Venice Area. "Atmospheric Environment", in press.

(4) Mattioli F., Tibaldi S., Pellizzarid P., 1974. - 1 Program for auto and cross spectral analysis. "L'Elaborazione Automatica". 1, 5, p. 44. 